\title{
Advanced healthcare for children and youth: application of targeted prevention, predictive diagnostics and personalised treatment approaches
}

\author{
Maurizio Scarpa \\ Published online: 6 July 2011 \\ (C) European Association for Predictive, Preventive and Personalised Medicine 2011
}

"The right treatment for the patient at the right moment at the highest efficacy" this is the dogma for the future of medicine, in particular, for the paediatric population. By years children have been excluded by the pharmaceutical developmental process having no or very late access to innovative drugs; to date bringing advancement from the translational medicine to children presents additional obstacles and requires specific efforts. Early diagnosis, search for biomarkers to understand pathophysiology and test the efficacy and safety of new treatments are the major goals to realise personalised medicines.

The increasing introduction of translational approaches, using biomarkers, has the potential of early identify patients cohorts, to increase the manageability of clinical trials (CT) possibly reducing their size, duration and cost. These results would be particularly advantageous in case of small populations where the standard CT model is difficult to apply.

This issue does address important topics targeted by the Personalised Medicine community and state the increasing interest in this field.

Clinical research is developing at an astonishing rate.

New molecules and drugs are being identified and are being progressed into clinical trials, whilst other compounds, which are clinically available, are being explored in different therapeutic indications or as combination therapy.

For many years the traditional approach to diagnosis and treatment has been based on symptoms and signs, which reflect, in the majority of the cases, the patient phenotype;

\footnotetext{
M. Scarpa $(\bowtie)$

Department of Paediatrics, University of Padova,

Padova, Italy

e-mail: maurizio.scarpa@unipd.it

M. Scarpa

Center for Rare Diseases Casa Sollievo della Sofferenza,

Scientific Institute and Hospital,

San Giovanni Rotondo, Foggia, Italy
}

trials have been conducted by grouping patients into broad groups with similar symptoms.

This, however, it is not enough anymore. Availability of new drugs, the advancement of knowledge of pathophysiologic aspect of a growing number of disorders and the evidence that early treatment is a must to obtain the most effective results of a therapy, do impose the development of strategies to define diagnosis as early as possible. For this reason, discussion at social and economical levels are ongoing to define for which disorder newborn screening must be offered. Newborn screening programmes will be of paramount impact for those disorders having an acute and life threatening event (mostly metabolic) which might be modified and mitigate by an early recognition.

However, discussions and debates are ongoing about those disorders which might have different grade of severity and a late onset. This will be facilitated by the discovery of biomarkers which will predict the biological progression of the disorder long before the phenotypic expression of it. Omics-based technologies will play a major role in producing early and, possibly, specific markers to be used to prevent permanent alterations.

These markers will be also of great importance to determine the tolerability, the safety and the efficacy of the new drugs and will have a major role in designing new and more effective clinical trials.

New concept of clinical trials is needed especially for the pediatric population and other small population disorders. Children, in fact, cannot be just considered as "small men".

So far, pharmaceutical and biotechnology companies have developed medicines for broad populations, and the regulatory assessment of risk and benefit has been based on the average clinical response across these groups.

Despite relevant advances in drug formulation, regulators, companies and clinicians have always known that not all patients will respond in the same way to the same therapy and that subpopulations with different degree of responsiveness 
should exist. However, the current methodological approach, based on well-designed randomised controlled CT, are not sufficient to identify, early in the developmental phase, which patient subpopulations are likely/unlikely to benefit from an experimental drugs. This leads to treat all patients at the same way while a clinical approach based on the subpopulations identification and stratification could be more efficient and of increased benefit for the patients.

In fact, the capacity to 'predict' the drug response both regarding efficacy and safety, could really change the patient perspective to be cured.

The paediatric population has a reduced access to innovative drugs and physicians often have to prescribe medicinal products authorised for adults in off-label way. The lack of paediatric medicines is the consequence of the lack of appropriate paediatric studies.

The conduct of clinical trials in children, despite representing a fundamental step to an effective and safe utilisation of drugs in the paediatric population, poses several problems. The difficulties lie in primis to the fact that, being the development of medicinal products for children intended for a small market, the pharmaceutical industry has always shown a little interest in the development of such drugs.

In addition, there are methodological issues mainly related to the difficulty in managing paediatric studies and to the small samples available which make paediatric studies limited and not supported yet by an adequate statistic power.

Personalised medicine, based on the premise: "the right treatment for the right patient at the right time" and in particular the integrated use of biomarkers as predictors of treatment response should be of particular interest for children.

At this regard, we should consider that the effects of the genetic polymorphism affecting the different actions the drugs may have on individuals at any age, can be considerably increased by the fact that growth and development, specific of the paediatric populations, lead to a larger variability in metabolising capabilities and, on the consequence, in drug response.

All these reasons have convinced The EPMA Journal to promote an Issue on Preventive Predictive and Personalised Medicine in Paediatrics, in order to highlight the need to address particular attention to children and adolescents. These are specific populations which deserve to have their own disorders treated with a customised therapy.

This issue is proposing a collection of 11 papers from 6 contributing countries (Fig. 1) highlighting important aspects of prevention, prediction and personalised medicine in Paediatrics. As discussed by M. Ozgüç and M. Dundar et al., new generation sequencing and highthroughput technologies are in progress to detect a wide number of disorders by the analysis of the whole genome (M. Ozgüç: Genetic testing: predictive value of genotyping for diagnosis and management of disease; M. Dundar et al.: Prediction, prevention and personalisation of medication for the prenatal period: genetic prenatal tests for both rare and common diseases). Identification of affected patients and carriers is essential for the modification of the natural history of genetic disorders and a more appropriate management of the disease. Prevention and prediction, however, might require also identification of the disorder before birth. This is described in a series of papers by the group of G.T. Tsangaris which are discussing new progresses on the utility of new biomarkers in the diagnosis and potential treatment of fetal abnormalities and pregnancy complications. Biomakers will be useful for the detection of abnormalities even in the maternal plasma, where cell free circulating foetal DNA can even be isolated, avoiding any need to collect amniotic fluid, preventing in this method any
Fig. 1 Map showing the countries of the contributors to the editorial and review articles in the current journal issue

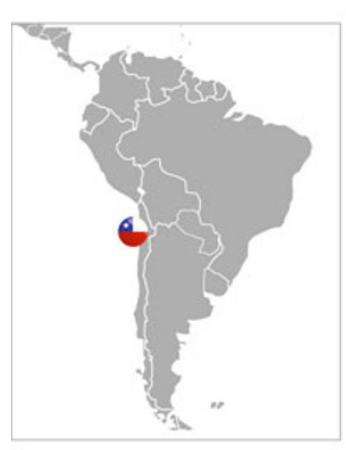

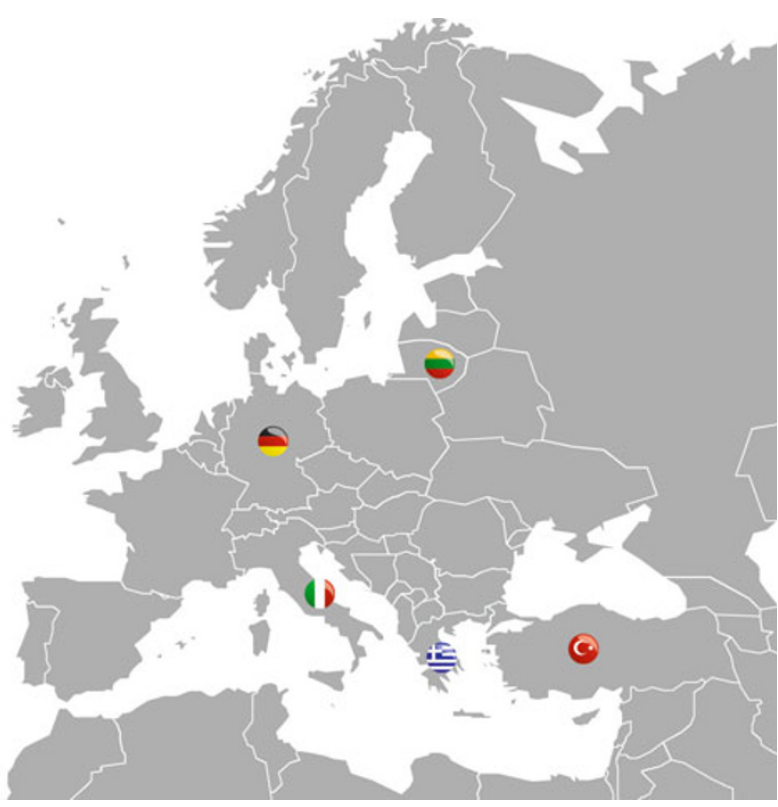


risk to the pregnancy (G.T. Tsangaris et al.: Application of proteomics for the identification of biomarkers in amniotic fluid: are we ready to provide a reliable prediction?; A. Kolialexi et al.: Biomarker development for non-invasive prenatal diagnosis of fetal aneuploidies: predictive reliability and potential clinical application; G. Tounta et al.: Noninvasive prenatal diagnosis using cell-free fetal nucleic acids in maternal plasma: Progress overview beyond predictive and personalized diagnosis).

New insights are proposed regarding a major problem affecting 1-2/1,000 newborns which is represented by the perinatal asphyxia. Search for biomarkers is essential in this field since prognosis about outcomes of hypoxic lesions. After asphyxia, infants can suffer from short to long-term neurological sequelae, their severity depend upon the extent of the insult, the metabolic imbalance during the reoxygenation period and the developmental state of the affected region. This issue is discussed in two papers highlighting the risk of hypoxic events secondary also to environmental and socio-economic circumstances (urban vs rural, work attitude etc.) and the recent achievements on biomarker search to understand the prognosis of perinatal asphyxia (O. Golubnitschaja et al.: Birth asphyxia as the major complication in newborns: moving towards improved individual outcomes by prediction, targeted prevention and tailored medical care; P. Morales et al.: Pathophysiology of perinatal asphyxia: can we predict and improve individual outcomes?).

The availability of new markers, the improvement of diagnosis and the development of treatments exploiting new biomarkers relevant to predict the involvement of CNS are changing the natural history of this life-threatening event.

Prevention, prediction and personalisation of medicine are a major goal of neurological disorders also in the field of rare diseases which alone affect $3-10 \%$ of the population.

The paper by M. Scarpa et al. describe a very interesting group of disorders, the lysosomal storage diseases, which in the last 10 years have attracted the attention of metabolic specialists and researchers. In fact, lysosome seems not to be anymore considered just the cell junk repository but rather the beating heart of the cell which is at the center of a complex system to ensure correct metabolic functions. In the last years the study of lysosomal function seems to be relevant not only to characterise lethal neurodegenerative disorders affecting 1:5,000 children (lysosomal storage diseases) but also for the understanding of the pathogenesis of more frequent adult disorders such as Parkinson and Alzheimer diseases (M. Scarpa et al.: Personalised medicine in paediatrics: individualising treatment in children with rare neurological diseases).

As it is clearly argued by A. Dietz and G. Wichmann, the absolute majority of otolaryngological diseases should be diagnosed and treated early in life (A. Dietz and G. Wichmann: Head and neck cancer: effective prevention in youth and predictive diagnostics for personalised treatment strategies according to biological differences). Ethical considerations are highlighted by E. Gefenas et al. (Does the "new philosophy" in predictive, preventive and personalised medicine require new ethics?).

This is just the beginning, a lot still need to be discussed.

We encourage readers to contribute with papers about paediatric personalised medicine research.

Paediatric needs are extremely wide and we want to maintain the attention very high.

Invited reviews and papers relevant to the discussion on important topics, such as: disorders of preterm and term infants, cancer, metabolic disorders, central nervous system, cardiology or asthma etc., the use of "OMICS" in childhood diseases, the organisation of biobanks and databases, the design of new clinical trials exploiting the Personalised Medicine dogma, will be welcome.

\section{Scarpa 05-2011}

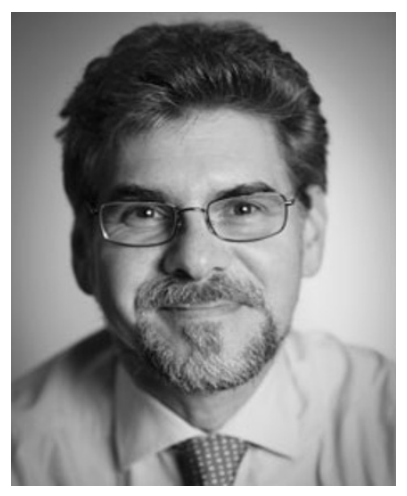

Maurizio Scarpa is the Head of the Lysosomal Unit at the Department of Paediatrics, University of Padova, and the Director of the Centre for Rare Disorders at the Casa Sollievo della Sofferenza Scientific Institute and Hospital at San Giovanni Rotondo (Foggia) Italy. He received his medical degree and doctorate from the University of Padova Medical School, Padova, and went on to specialise in paediatrics at the same institution. He completed a postdoctoral fellowship at the European Molecular Biology Laboratory (EMBL) Heidelberg, Germany, on Molecular Biology and Gene Expression and at the Howard Hughes Medical Institute, Institute for Molecular Genetics, Baylor College of Medicine, Houston, TX, USA, on genetics and gene therapy. He then was appointed as consultant on Molecular Biology at the Middlesex Hospital and the London Hospital in London, UK.

Dr. Scarpa is the Director of the Genetics and Biochemistry PhD Programme at the Department of Paediatrics, University of Padova, Italy. $\mathrm{He}$ is the Director of the Office of Foreign Affairs of the University of Padova Medical School.

Dr. Scarpa has vast clinical experience in the diagnosis and treatment of lysosomal storage disorders (LSDs) and neurometabolic diseases, and is especially interested in developing diagnostic assays and new approaches for the treatment of LSDs.

Currently, Dr. Scarpa is coordinating the SHIRE CNS Working Group and, together with Dr. David Begley King's College of London UK, he is the founder and Scientific Coordinator of the "BRAINS FOR BRAIN" Research Consortium a Paneuropean Task Force on Brain and Neurodegenerative Lysosomal Storage Diseases. Currently, he is the Vice president of the BRAINS FOR BRAIN FOUNDATION (www.brains4brain.eu). 